\title{
LiteBIRD: A satellite for the studies of B-mode polarization and inflation from cosmic background radiation detection
}

\author{
M. Hazumi ${ }^{19,22,29,49}$ - P.A.R. Ade ${ }^{47}$ - Y. Akiba ${ }^{19,49}$. \\ D. Alonso ${ }^{41}$ - K. Arnold ${ }^{16}$ - J. Aumont ${ }^{20}$. \\ C. Baccigalupi ${ }^{25}$. D. Barron ${ }^{48}$. S. Basak ${ }^{11,25}$. \\ S. Beckman ${ }^{15}$. J. Borrill ${ }^{6,48}$. F. Boulanger ${ }^{20}$. \\ M. Bucher ${ }^{3}$. E. Calabrese C $^{47}$. Y. Chinone ${ }^{15,29}$. \\ S. Cho $^{13}$ - A. Cukierman ${ }^{15}$ - D.W. Curtis ${ }^{48}$. \\ T. de Haan $^{43}$. M. Dobbs ${ }^{42}$. A. Dominjon ${ }^{34}$. \\ T. Dotani ${ }^{22}$. L. Duband ${ }^{18}$. A. Ducout ${ }^{29}$. \\ J. Dunkley ${ }^{10,41}$ · J.M. Duval ${ }^{18}$. T. Elleflot ${ }^{16}$. \\ H.K. Eriksen ${ }^{24}$ · J. Errard ${ }^{3}$ · J. Fischer ${ }^{48}$. \\ T. Fujino ${ }^{53}$. T. Funaki ${ }^{12}$. U. Fuskeland ${ }^{24}$. \\ K. Ganga ${ }^{3}$ - N. Goeckner-Wald ${ }^{15}$. J. Grain ${ }^{20}$. \\ N.W. Halverson ${ }^{4,9,17}$. T. Hamada ${ }^{2,19}$. \\ T. Hasebe ${ }^{22}$. M. Hasegawa ${ }^{19,49} \cdot$ K. Hattori ${ }^{36}$. \\ M. Hattori ${ }^{2}$. L. Hayes ${ }^{48}$. N. Hidehira ${ }^{12}$. \\ C.A. Hill ${ }^{15,43}$. G. Hilton ${ }^{38}$. J. Hubmayr ${ }^{38}$. \\ K. Ichiki ${ }^{31}$. T. Iida $^{29}$ - H. Imada Im. Inoue $^{39}$. \\ Y. Inoue ${ }^{19,21}$ - K.D. Irwin ${ }^{13,28}$ - H. Ishino ${ }^{12}$. \\ O. Jeong ${ }^{15}$ - H. Kanai ${ }^{53}$. D. Kaneko ${ }^{29}$. \\ S. Kashima ${ }^{34}$. N. Katayama ${ }^{29}$. T. Kawasaki ${ }^{30}$. \\ S.A. Kernasovskiy ${ }^{13}$. R. Keskitalo ${ }^{6,48}$. \\ A. Kibayashi ${ }^{12}$. Y. Kida ${ }^{12}$. K. Kimura ${ }^{39}$ \\ T. Kisner ${ }^{6,48}$ - K. Kohri ${ }^{19}$. E. Komatsu ${ }^{33}$. \\ K. Komatsu ${ }^{12}$ - C.L. Kuo ${ }^{13,28}$. N.A. Kurinsky ${ }^{13,28}$

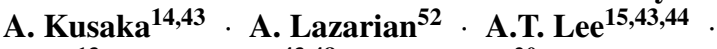 \\ D. $\mathrm{Li}^{13}$. E. Linder ${ }^{43,48}$. B. Maffei ${ }^{20}$ \\ A. Mangilli $^{20}$. M. Maki ${ }^{19}$. T. Matsumura ${ }^{29}$. \\ S. Matsuura ${ }^{26}$. D. Meilhan ${ }^{48}$. S. Mima ${ }^{45}$. \\ Y. Minami ${ }^{19}$. K. Mitsuda ${ }^{22}$. L. Montier ${ }^{5}$. \\ M. Nagai ${ }^{34}$. T. Nagasaki ${ }^{19}$. R. Nagata ${ }^{19}$. \\ M. Nakajima ${ }^{39}$. S. Nakamura ${ }^{53}$. T. Namikawa ${ }^{13}$. \\ M. Naruse ${ }^{46} \cdot$ H. Nishino ${ }^{19}$. T. Nitta ${ }^{51}$. \\ T. Noguchi $^{34}$ - H. Ogawa ${ }^{39}$ - S. Oguri ${ }^{45}$. \\ N. Okada ${ }^{23} \cdot$ A. Okamoto ${ }^{23}$. T. Okamura ${ }^{19}$ \\ C. Otani ${ }^{45}$ - G. Patanchon ${ }^{3}$ - G. Pisano ${ }^{47}$. \\ G. Rebeiz $^{16}$ - M. Remazeilles ${ }^{50}$. P.L. Richards ${ }^{15}$. \\ S. Sakai ${ }^{22}$. Y. Sakurai ${ }^{29}$. Y. Sato ${ }^{23}$. N. Sato ${ }^{19}$. \\ M. Sawada ${ }^{1}$. Y. Segawa ${ }^{19,49}$. Y. Sekimoto ${ }^{8,22,49}$ \\ U. Seljak $^{15}$ - B.D. Sherwin ${ }^{7,27,43}$. T. Shimizu ${ }^{8}$. \\ K. Shinozaki ${ }^{23}$. R. Stompor ${ }^{3}$. H. Sugai ${ }^{29}$. \\ H. Sugita $^{23}$. A. Suzuki ${ }^{15,44}$. J. Suzuki ${ }^{19}$. \\ O. Tajima ${ }^{19,49}$. S. Takada ${ }^{35}$. R. Takaku ${ }^{53}$.
}



S. Takakura ${ }^{19,40}$ - S. Takatori ${ }^{19,49}$. D. Tanabe $\mathrm{T}^{19,49}$.
E. Taylor ${ }^{48}$. K.L. Thompson ${ }^{13,28}$. B. Thorne ${ }^{29,41}$.
T. Tomaru ${ }^{19}$ - T. Tomida ${ }^{22}$. N. Tomita ${ }^{14}$.

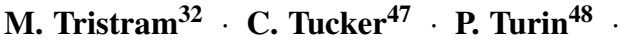
M. Tsujimoto ${ }^{22}$. S. Uozumi ${ }^{12}$. S. Utsunomiya ${ }^{29}$.
Y. Uzawa ${ }^{37}$. F. Vansyngel ${ }^{20}$. I.K. Wehus ${ }^{24}$.
B. Westbrook ${ }^{15}$. M. Willer ${ }^{48}$. N. Whitehorn ${ }^{15}$.
Y. Yamada ${ }^{12}$. R. Yamamoto Y $^{22}$. Yamasaki ${ }^{22}$.
T. Yamashita ${ }^{53}$. M. Yoshida ${ }^{19}$

the date of receipt and acceptance should be inserted later

E-mail: Masashi Hazumi: masashi.hazumi@kek.jp

${ }^{1}$ Aoyama Gakuin University, Sagamihara, Kanagawa 252-5258, Japan

${ }^{2}$ Astronomical Institute, Graduate School of Science, Tohoku University, Sendai, 980-8578, Japan

${ }^{3}$ AstroParticule et Cosmologie (APC), Univ Paris Diderot, CNRS/IN2P3, CEA/Irfu, Obs de Paris, Sorbonne Paris Cité, France

${ }^{4}$ Center for Astrophysics and Space Astronomy, University of Colorado, Boulder, CO 80309, USA

${ }^{5}$ CNRS, IRAP, F-31028 Toulouse cedex 4, France

${ }^{6}$ Computational Cosmology Center, Lawrence Berkeley National Laboratory, Berkeley, CA 94720, USA

${ }^{7}$ DAMTP, University of Cambridge, Cambridge CB3 0WA, UK

${ }^{8}$ Department of Astronomy, The University of Tokyo, Tokyo 113-0033, Japan

${ }^{9}$ Department of Astrophysical and Planetary Sciences, University of Colorado, Boulder, CO 80309, USA

${ }^{10}$ Department of Astrophysical Sciences, Princeton University, Princeton, NJ 08544, USA

${ }^{11}$ School of Physics, Indian Institute of Science Education and Research Thiruvananthapuram, Maruthamala PO, Vithura, Thiruvananthapuram 695551, Kerala, India

${ }^{12}$ Department of Physics, Okayama University, Okayama, Okayama 700-8530, Japan

${ }^{13}$ Department of Physics, Stanford University, Stanford, CA 94305-4060, USA

${ }^{14}$ Department of Physics, The University of Tokyo, Tokyo 113-0033, Japan

${ }^{15}$ Department of Physics, University of California, Berkeley, CA 94720, USA

${ }^{16}$ Department of Physics, University of California, San Diego, CA 92093-0424, USA

${ }^{17}$ Department of Physics, University of Colorado, Boulder, CO 80309, USA

${ }^{18}$ French Alternative Energies and Atomic Energy Commission (CEA), Grenoble, France

${ }^{19}$ High Energy Accelerator Research Organization (KEK), Tsukuba, Ibaraki 305-0801, Japan

${ }^{20}$ Institut d'Astrophysique Spatiale (IAS), CNRS, UMR 8617, Université Paris-Sud 11, Bâtiment 121, 91405 Orsay, France 
${ }^{21}$ Institute of Physics, Academia Sinica, 128, Sec.2, Academia Road, Nankang, Taiwan

${ }^{22}$ Institute of Space and Astronautical Science (ISAS), Japan Aerospace Exploration Agency (JAXA), Sagamihara, Kanagawa 252-5210, Japan

${ }^{23}$ Research and Development Directorate, Japan Aerospace Exploration Agency (JAXA), Tsukuba, Ibaraki 305-8505, Japan

${ }^{24}$ Institute of Theoretical Astrophysics, University of Oslo, NO-0315 Oslo, Norway

${ }^{25}$ International School for Advanced Studies (SISSA), Via Bonomea 265, 34136, Trieste, Italy

${ }^{26}$ Kansei Gakuin University, Nishinomiya, Hyogo 662-8501, Japan

${ }^{27}$ Kavli Institute for Cosmology Cambridge, Cambridge CB3 OHA, UK

${ }^{28}$ Kavli Institute for Particle Astrophysics and Cosmology (KIPAC), SLAC National Accelerator Laboratory, Menlo Park, CA 94025, USA

${ }^{29}$ Kavli Institute for the Physics and Mathematics of the Universe (Kavli IPMU, WPI), UTIAS, The University of Tokyo, Kashiwa, Chiba 277-8583, Japan

${ }^{30}$ Kitazato University, Sagamihara, Kanagawa 252-0373, Japan

${ }^{31}$ Kobayashi-Maskawa Institute for the Origin of Particle and the Universe, Nagoya University, Nagoya, Aichi 464-8602, Japan

${ }^{32}$ Laboratoire de l'Accélérateur Linéaire (LAL), Univ. Paris-Sud, CNRS/IN2P3, Université Paris-Saclay, Orsay, France

${ }^{33}$ Max-Planck-Institut for Astrophysics, D-85741 Garching, Germany

${ }^{34}$ National Astronomical Observatory of Japan (NAOJ), Mitaka, Tokyo 181-8588, Japan

${ }^{35}$ National Institute for Fusion Science (NIFS), Toki, Gifu 509-5202, Japan

${ }^{36}$ National Institute of Advanced Industrial Science and Technology (AIST), Tsukuba, Ibaraki 305-8563, Japan

${ }^{37}$ National Institute of Information and Communications Technology (NICT), Kobe, Hyogo 651-2492, Japan

${ }^{38}$ National Institute of Standards and Technology (NIST), Boulder, Colorado 80305, USA

${ }^{39}$ Osaka Prefecture University, Sakai, Osaka 599-8531, Japan

${ }^{40}$ Osaka University, Toyonaka, Osaka 560-0043, Japan

${ }^{41}$ Oxford Astrophysics, Oxford, OX1 3RH, United Kingdom

${ }^{42}$ Physics Department, McGill University, Montreal, QC H3A 0G4, Canada

${ }^{43}$ Physics Division, Lawrence Berkeley National Laboratory, Berkeley, CA 94720, USA

${ }^{44}$ Radio Astronomy Laboratory, University of California, Berkeley, CA 94720, USA

${ }^{45}$ RIKEN, Wako, Saitama 351-0198, Japan

${ }^{46}$ Saitama University, Saitama, Saitama 338-8570, Japan

${ }^{47}$ School of Physics and Astronomy, Cardiff University, Cardiff CF10 3XQ, United Kingdom 


\begin{abstract}
LiteBIRD is a candidate satellite for a strategic large mission of JAXA. With its expected launch in the middle of 2020's with a H3 rocket, LiteBIRD plans to map the polarization of the cosmic microwave background $(\mathrm{CMB})$ radiation over the full sky with unprecedented precision. The full success of LiteBIRD is to achieve $\delta r<0.001$, where $\delta r$ is the total error on the tensor-to-scalar ratio $r$. The required angular coverage corresponds to $2 \leq \ell \leq 200$, where $\ell$ is the multipole moment. This allows us to test well-motivated cosmic inflation models. Full-sky surveys for three years at a Lagrangian point L2 will be carried out for 15 frequency bands between 34 and $448 \mathrm{GHz}$ with two telescopes to achieve the total sensitivity of $2.5 \mu \mathrm{K}$-arcmin with a typical angular resolution of $0.5^{\circ}$ at $150 \mathrm{GHz}$. Each telescope is equipped with a half-wave plate system for polarization signal modulation and a focal plane filled with polarization-sensitive TES bolometers. A cryogenic system provides a $100 \mathrm{mK}$ base temperature for the focal planes and $2 \mathrm{~K}$ and $5 \mathrm{~K}$ stages for optical components.
\end{abstract}

Keywords Cosmic Inflation, Cosmic Microwave Background, B-Mode Polarization, Primordial Gravitational Wave, Quantum Gravity, Satellite

\title{
1 Introduction
}

Although the Big Bang theory has been tested well by many observations, there are a number of critical problems that remain unanswered. The leading theory today to resolve the problems is cosmological inflation, which hypothesizes that our universe went through an accelerating expansion at the very early stage before the hot Big Bang.

The cosmic inflation predicts that primordial gravitational waves were created during the inflationary era. The primordial gravitational waves should have imprinted a special signature, called the B-mode, in the polarization patterns of the CMB. ${ }^{1,2,3,4}$ Measurements of the polarization of the $\mathrm{CMB}$ are known as the most sensitive probe for the primordial gravitational waves. Yet a state-of-the-art technology is required for detection, since the B-mode signal is expected to be much fainter than the ordinary pattern called E-mode.

LiteBIRD is a satellite for stringent tests of the cosmic inflation by performing a fullsky CMB polarization survey at degree scales. In JAXA's strategic large mission program for space science, three satellites are planned to be launched in the next 10 years. A launch window in the middle of 2020's is the target for LiteBIRD. After passing the initial selection, LiteBIRD is currently one of two candidates in Phase-A1 of Institute of Space and Astronautical Science (ISAS) at JAXA for concept development. A global joint study group has been formed with researchers from Japan, U.S., Canada and Europe to carry out Phase-A1 studies. The final selection will take place in 2019.

\footnotetext{
${ }^{48}$ Space Sciences Laboratory, University of California, Berkeley, CA 94720, USA

${ }^{49}$ The Graduate University for Advanced Studies (SOKENDAI), Miura District, Kanagawa 240-0115, Hayama, Japan

${ }^{50}$ The University of Manchester, Manchester M13 9PL, United Kingdom

${ }^{51}$ Division of Physics, Faculty of Pure and Applied Sciences, University of Tsukuba, Ibaraki 305-8571, Japan

${ }^{52}$ University of Wisconsin-Madison, Madison, Wisconsin 53706, USA

${ }^{53}$ Yokohama National University, Yokohama, Kanagawa 240-8501, Japan
} 


\section{Science Goals}

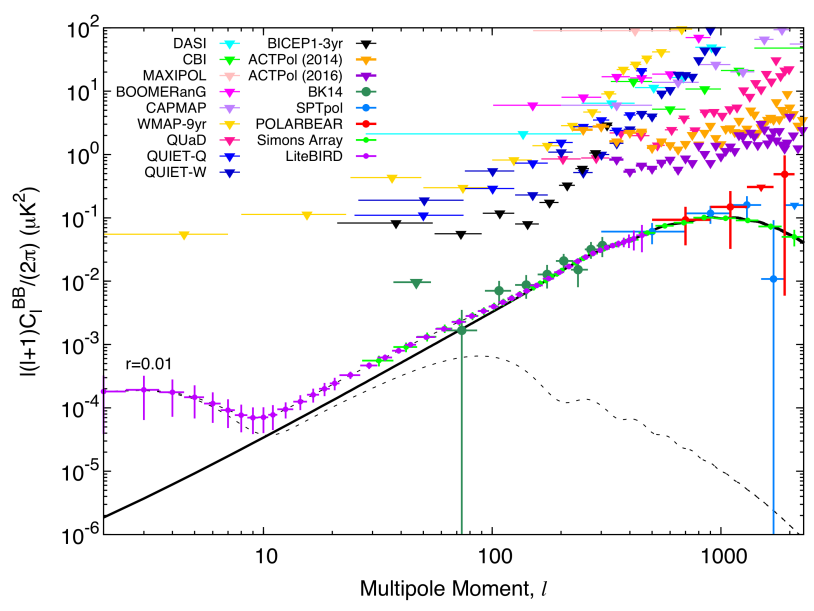

Fig. 1 Summary of present measurements of CMB B-mode power spectra and expected sensitivity of LiteBIRD. Circles with vertical bars show central values and errors, while triangles are upper limits.

The present measurements of CMB B-mode power spectra are summarized in Fig. 1, with the expected sensitivities of LiteBIRD. The B-mode power is proportional to the tensorto-scalar ratio, $r$, which is observationally constrained to be $r<0.07^{5}$. The next-generation CMB polarization experiments may see a hint at the signal around $\ell \sim 100$, which is due to recombination. If $r$ is less than $\sim 0.03$, however, the B-mode due to gravitational lensing becomes dominant. Removing contamination of the lensing B-mode, often called "delensing", is needed in this case. In contrast, another excess in $\ell<10$, which is due to reionization, is larger than the lensing B-mode even at $r=0.001$. In order to access the reionization peak, one needs to survey the full sky, where the advantage of observations in space is clear.

The full success of LiteBIRD is to achieve $\delta r<0.001$ without delensing, for a multiple range $2 \leq \ell \leq 200$. Here $\delta r$ is the total uncertainty on $r$, which consists of five components: (instrumental) statistical uncertainties; systematic uncertainties; uncertainties due to contamination of foreground components; uncertainties due to gravitational lensing; uncertainties due to observer biases. Many inflationary models predict $r>0.01$, for which LiteBIRD will provide more than $10 \sigma$ statistical significance. If LiteBIRD finds no primordial B-mode, an upper limit of $r<0.002$ at $95 \%$ C.L. will be obtained. This will exclude many well-motivated inflationary models, and give a severe constraint on physics of inflation. If some indication of the primordial B-mode is obtained prior to observations by LiteBIRD, it implies a fairly large value of $r$. In this case, data from LiteBIRD will allow us to measure the B-mode signals from reionization and recombination simultaneously. If the spectral shape is consistent with the expectation from the standard cosmology, it will drastically narrow down the list of possible inflationary models, and provide a much deeper insight on the correct model of inflation. If we observe a totally unexpected power spectrum beyond the standard model, that will lead to revolution in our picture of the universe. 
In any case described above, the measurement of the primordial B-mode at LiteBIRD will also be the first stringent test of quantum gravity, which should exist behind any inflationary model. Here quantum gravity means a theory that copes with two pillars of physics, 1) Einstein's theory of general relativity that describes gravity, and 2) quantum physics, in a single framework.

To achieve the full success, our uncertainty budget is defined such that an equal amount, $(1 / \sqrt{3}) \times 10^{-3}=0.57 \times 10^{-3}$, is given to each of the following three components; the total statistical uncertainty after the foreground separation $\sigma_{\text {stat }}$, the total systematic uncertainty $\sigma_{\text {syst }}$, and a margin. The requirements are thus $\sigma_{\text {stat }}<0.57 \times 10^{-3}$ and $\sigma_{\text {syst }}<0.57 \times 10^{-3}$. Since we assume no delensing with external data, $\sigma_{\text {stat }}$ includes those from the lensing Bmode component and additional uncertainties due to foreground separation. The observer bias needs to be much smaller than $\sigma_{\text {syst }}$.

\section{System Overview}

Table 1 shows baseline specifications of LiteBIRD, which were adopted at the beginning of the Phase-A1 concept development in 2016, except that the orbit and scan strategy have been modified in 2017. JAXA's H3 rocket will be used to launch and put LiteBIRD into an

\begin{tabular}{|c|l|}
\hline \multicolumn{1}{|c|}{ Item } & Specification \\
\hline \hline Launch year & $2026-2027$ \\
\hline Launch vehicle & JAXA H3 \\
\hline Observation type & All-sky CMB surveys \\
\hline Observation time & 3 years \\
\hline Orbit & L2 Lissajous orbit \\
\hline Scan strategy & Spin and precession $\left(\alpha=45^{\circ}, \beta=50^{\circ}\right)$ \\
\hline Observing frequencies & $34-448 \mathrm{GHz}$ \\
\hline Number of bands & 15 \\
\hline Sensitivity & $2.5 \mu \mathrm{K}$-arcmin $(3$ years $)$ \\
\hline Angular resolution & $0.5^{\circ}$ at $100 \mathrm{GHz}($ FWHM) \\
\hline Mission instruments & $\cdot$ Superconducting detector arrays \\
& $\cdot$ Polarization modulator with continously-rotating half-wave plate (HWP) \\
& $\cdot$ Crossed-Dragone mirrors (LFT) + small refractive telescope (HFT) \\
\hline Data size & 4 GB/day \\
\hline Mass & $2.2 \mathrm{t}$ \\
\hline Power & $2.5 \mathrm{~kW}$ \\
\hline
\end{tabular}

Table 1 Main specifications of LiteBIRD. Parameters are from the Phase-A 2016 baseline, except the orbit and the scan strategy.

orbit around one of Sun-earth Lagrangian points known as L2 for full-sky surveys for three years. A Lissajous orbit is our new choice due mainly to the better thermal condition than halo orbits. In our scan strategy (Fig. 2), the spacecraft spins about the spin axis at $0.1 \mathrm{rpm}$. The spin axis itself also rotates but at a much slower rate; the current studies range from 90 minutes to nearly a day. The spin axis is canted $\alpha=45^{\circ}$ off the Sun-L2 axis. The angle $\beta$ between the boresight and the spin axis is $50^{\circ}$.

The requirements on the scan strategy include good thermal stability, good uniformity on the moving direction of boresight pointing across each sky pixel ("attack angle" uniformity), 


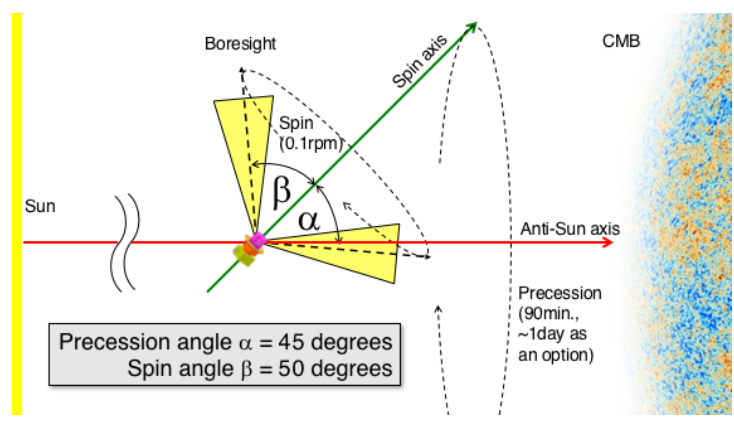

Fig. 2 Scan strategy of LiteBIRD in a Lissajous orbit around L2.

good observation uniformity on sky pixels, large daily sky coverage, and short revisit times for each sky pixel. These are important to mitigate instrumental systematic uncertainties.

A typical angular resolution is $30 \mathrm{arcmin}$ at $150 \mathrm{GHz}$, which is sufficient to detect both reionization and recombination peaks of the B-mode power spectrum. We require measurements with 15 frequency bands between 34 and $448 \mathrm{GHz}$ to achieve the total sensitivity of $2.5 \mu \mathrm{K}$-arcmin with three years of observation. The lensing B-mode behaves as the external noise unless delensing is applied. It is therefore important that the total sensitivity is well below the lensing "floor", which is approximately at $5 \mu \mathrm{K}$-arcmin. The main reason for requiring 15 frequency bands is foreground separation. Detailed foreground separation studies already show promising results ${ }^{6}$.

Figure 3 shows an overview of the baseline mission payload design of LiteBIRD defined at the beginning of Phase-A1, which is called the 2016 baseline hereafter. Since the goal of Phase-A1 is to improve the design through various tradeoff studies, the final configuration at the end of Phase-A1 may look different. Nevertheless the 2016 baseline design has all the key features one needs to consider.
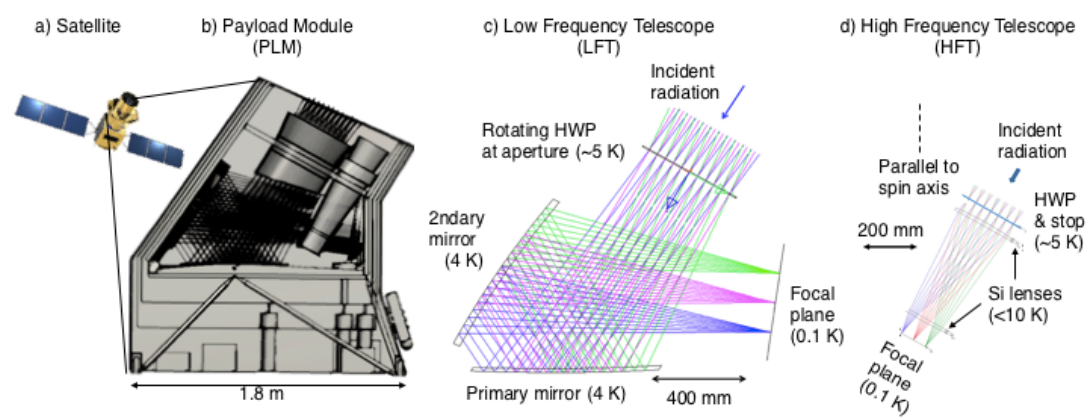

Fig. 3 Conceptual design of LiteBIRD. The payload module (PLM) houses a low frequency telescope (LFT) and a high frequency telescope (HFT).

There are two telescopes, each equipped with a continuously-rotating half-wave plate (HWP) system for polarization signal modulation and a focal plane filled with polarizationsensitive TES bolometers. A cryogenic system provides a $100 \mathrm{mK}$ base temperature for the focal planes and $2 \mathrm{~K}$ and $5 \mathrm{~K}$ stages for optical components. We will use the crossed Mizuguchi-Dragone configuration for the low frequency telescope (LFT). The diameter of 
the primary mirror is about $80 \mathrm{~cm}$. In order to mitigate sidelobes sufficiently, we place a cold stop at the sky side of the primary mirror, which determines the aperture. The aperture diameter is $40 \mathrm{~cm}$. We will adopt a continuously-rotating HWP with a diameter of $45 \mathrm{~cm}$ and a spin rate of about $1 \mathrm{~Hz}$ at the most sky side near the cold stop. Since the HWP will also be cooled below $10 \mathrm{~K}$, we plan to use superconducting magnetic bearing for the HWP rotator. More details on our HWP development are shown elsewhere ${ }^{7}$. The focal plane detector

\begin{tabular}{|r|r|r|r|r|r|r|r|r|}
\hline $\begin{array}{r}\text { Freq. } \\
(\mathrm{GHz})\end{array}$ & $\begin{array}{r}\theta_{\text {FWHM }} \\
(\operatorname{arcmin})\end{array}$ & $\Delta v / v$ & $\begin{array}{r}\text { Pixel } \\
\text { size } \\
(\mathrm{mm})\end{array}$ & $N_{\text {det }}$ & $\begin{array}{r}\mathrm{NET}_{\text {det }} \\
(\mu \mathrm{K} \sqrt{s})\end{array}$ & $\begin{array}{r}\mathrm{NET}_{\text {array }}^{T} \\
(\mu \mathrm{K} \sqrt{s})\end{array}$ & $\begin{array}{r}\mathrm{NET}_{\text {array }}^{P} \\
(\mu \mathrm{K} \sqrt{s})\end{array}$ & $\begin{array}{r}\omega_{P}^{-1 / 2} \\
(\mu \mathrm{K} \text {-arcmin })\end{array}$ \\
\hline 40 & 69 & 0.30 & 18.0 & 114 & 172 & 18.0 & 25.5 & 37.5 \\
50 & 56 & 0.30 & 18.0 & 114 & 96 & 11.5 & 16.3 & 24.0 \\
60 & 48 & 0.23 & 18.0 & 114 & 79 & 9.5 & 13.5 & 19.9 \\
68 & 43 & 0.23 & 18.0 & 114 & 64 & 7.8 & 11.0 & 16.2 \\
78 & 39 & 0.23 & 18.0 & 114 & 54 & 6.5 & 9.2 & 13.5 \\
89 & 35 & 0.23 & 18.0 & 114 & 47 & 5.6 & 8.0 & 11.7 \\
\hline 100 & 29 & 0.23 & 12.0 & 296 & 59 & 4.4 & 6.2 & 9.2 \\
119 & 25 & 0.30 & 12.0 & 222 & 42 & 3.7 & 5.2 & 7.6 \\
140 & 23 & 0.30 & 12.0 & 296 & 38 & 2.8 & 4.0 & 5.9 \\
166 & 21 & 0.30 & 12.0 & 222 & 36 & 3.1 & 4.4 & 6.5 \\
195 & 20 & 0.30 & 12.0 & 296 & 37 & 2.8 & 3.9 & 5.8 \\
235 & 19 & 0.30 & 12.0 & 222 & 43 & 3.7 & 5.2 & 7.7 \\
\hline 280 & 24 & 0.30 & 5.4 & 128 & 55 & 6.3 & 8.9 & 13.2 \\
337 & 20 & 0.30 & 4.5 & 128 & 81 & 9.4 & 13.2 & 19.5 \\
402 & 17 & 0.23 & 4.0 & 128 & 156 & 18.0 & 25.5 & 37.5 \\
\hline Total & & & & 2622 & & 1.2 & 1.7 & 2.5 \\
\hline
\end{tabular}

Table 2 Focal plane parameters of the 2016 baseline design of LiteBIRD. In the calculation, the aperture stop temperature is $2 \mathrm{~K}$, mirrors for LFT and lenses for HFT are at $5 \mathrm{~K}$. The NET values include a margin (13\%), and the expected noise on the polarization signal on a sky pixel $\left(\omega_{P}^{-1 / 2}\right)$ takes into account the end-to-end detector/readout yield of $80 \%$, and inefficiencies due to cosmic ray hits (15\%) and ADR recycling (15\%).

array for LFT is equipped with multi-chroic TES bolometers. Table 2 shows focal plane parameters for the 2016 baseline design, where LFT houses 12 bands with center frequencies of 40, 50, 60, 68, 78, 89, 100, 119, 140, 166, 195, $235 \mathrm{GHz}$. The design avoids CO lines with notch filters. Hardware implementation with multi-chroic TES technology is discussed elsewhere ${ }^{8}$. The number of detectors for each frequency is carefully chosen to achieve required sensitivities on $\mathrm{CMB}$ signals and sufficient foreground separation performance, and to keep the heat load from readout lines low enough. All detector pixels are placed within an area of high Strehl ratios greater than 0.95 .

In the 2016 baseline design, the high frequency telescope (HFT) adopts refractive optics with two lenses, and houses a detector wafer for 280,337 and $402 \mathrm{GHz}$ on a telecentric focal plane ${ }^{8}$. The aperture size is $20 \mathrm{~cm}$ and the field-of-view is $10 \times 10$ degrees $^{2}$. The refractive design is preferred because its small size is beneficial for cooling. In order to have more powerful foreground separation capability, design work is in progress to accommodate seven detector wafers. More details on such enhanced designs are described elsewhere ${ }^{9}$.

The LiteBIRD cooling system is rather similar to SPICA's pre-cooling system based on Stirling and JT coolers, and an ADR is very similar to one of future X-ray missions such as ATHENA. Evaluations of these instruments are ongoing under ESA's cryogenic-chain core technology program $(\mathrm{CC}-\mathrm{CTP})^{10}$. In order to minimize thermal contacts due to readout cables, we plan to use the digital active nulling (DAN) technique to multiplex 78 channels $^{8}$. 
The data size is estimated to be $4 \mathrm{~GB}$ /day with a loss-less data compression algorithm and including JAXA's standard data structure for telemetry. The next-generation telemetry system based on the X-band data links will be sufficient to download LiteBIRD data.

\section{Discussions}

The current design of LiteBIRD has good extendability. For example, we have considered an enhanced HFT with more detectors and frequency bands ${ }^{9}$. We have checked that the enhanced HFT design satisfies requirements from the cooling capability, the data link capability and the envelope of the $\mathrm{H} 3$ rocket. By choosing a proper pixel size, it is also possible to implement more detectors using two corners of the LFT focal plane which are not used but have high-enough Strehl ratios. Band centers can further be distributed to increase the effective number of bands in case needed.

Although we expect to have many outcomes in cosmology from CMB polarization maps of LiteBIRD, we focus on the full success, $\delta r<0.001$, in our system design. Advantages of having a focused mission are clear. Relatively small mirror sizes are adequate, which are better for cooling. The whole payload module can be tested in a large cryogenic test chamber. This will allow us to obtain better calibration data on ground, which will result in smaller systematic uncertainties. We can also perform better end-to-end pre-flight investigations, which will important to reduce the chance of failure.

The result on the $r$ measurement can further be improved by using external data for delensing and foreground separation. For example, delensing can be performed with future ground-based CMB measurements such as CMB-S4, or the SKA continuum survey ${ }^{11}$. Low-frequency measurements such as C-BASS and its upgrade are useful for foreground separation ${ }^{12}$. Any of these improvements is defined as the extra success of LiteBIRD.

\section{Conclusions}

With its expected launch in the middle of 2020's with JAXA's H3 rocket, LiteBIRD plans to map the CMB polarization over the full sky with unprecedented precision. The full success of LiteBIRD is to achieve $\delta r<0.001$ in a range $2 \leq \ell \leq 200$. This allows us to test well-motivated cosmic inflation models. In addition to the full success, LiteBIRD is also expected to produce various scientific outcomes in cosmology. A three-year full-sky survey at L2 will be carried out for 15 frequency bands between 34 and $448 \mathrm{GHz}$ with two telescopes to achieve the total sensitivity of $2.5 \mu \mathrm{K}$-arcmin with a typical angular resolution of $0.5^{\circ}$ at $150 \mathrm{GHz}$. Each telescope is equipped with a half-wave plate system for polarization signal modulation and a focal plane filled with polarization-sensitive multi-chroic TES bolometers. A cryogenic system provides a $100 \mathrm{mK}$ base temperature for the focal planes and $2 \mathrm{~K}$ and $5 \mathrm{~K}$ temperature stages for optical components. The current design has good extendability to add more detectors and frequency bands. Polarization-sensitive TES bolometers at LiteBIRD play a key role in addressing one of the most important problems in cosmology. They will also open up a new era of stringent tests of quantum gravity.

Acknowledgements This work was supported by the ISAS/JAXA Phase-A1 program, by MEXT KAKENHI Grant Numbers JP15H05891, 21111002, by World Premier International Research Center Initiative (WPI), MEXT, and by the JSPS Core-to-Core program. Development of the sub-Kelvin instrument was supported by the NASA mission of opportunity Phase A program. 


\section{References}

1. Kamionkowski, M., Kosowsky, A., and Stebbins, A., "A Probe of primordial gravity waves and vorticity," Phys.Rev.Lett. 78, 2058-2061 (1997).

2. Seljak, U. and Zaldarriaga, M., "Signature of gravity waves in polarization of the microwave background," Phys.Rev.Lett. 78, 2054-2057 (1997).

3. Zaldarriaga, M. and Seljak, U., "An all sky analysis of polarization in the microwave background," Phys.Rev. D55, 1830-1840 (1997).

4. Kamionkowski, M., Kosowsky, A., and Stebbins, A., "Statistics of cosmic microwave background polarization," Phys.Rev. D55, 7368-7388 (1997).

5. P. A. R. Ade et al. [BICEP2 and Keck Array Collaborations], Phys. Rev. Lett. 116, 031302 (2016)

6. M. Remazeilles, C. Dickinson, H. K. Eriksen and I. K. Wehus, arXiv:1707.02981 [astroph.CO].

7. T. Matsumura et al., "Development of a half-wave plate based polarization modulator unit for LiteBIRD”, Journal of Low Temperature Physics, This Special Issue, 2017, Submitted.

8. A. Suzuki et al. (LiteBIRD Joint Study Group), "The LiteBIRD Satellite Mission - SubKelvin Instrument”, Journal of Low Temperature Physics, This Special Issue, 2017, Submitted.

9. T. Hasebe et al. (LiteBIRD Joint Study Group), "Concept Design of High Frequency Telescope for LiteBIRD”, Journal of Low Temperature Physics, This Special Issue, 2017, Submitted.

10. N. Y. Yamasaki et al., "International Development of Detector Cooling System Down to $50 \mathrm{mK}$ in Space without Cryogens", Journal of Low Temperature Physics, This Special Issue, 2017, Submitted.

11. T. Namikawa, D. Yamauchi, B. Sherwin and R. Nagata, Phys. Rev. D 93, no. 4, 043527 (2016).

12. M. O. Irfan et al., MNRAS, Volume 448, Issue 4, 21 April 2015. 